\title{
MINERALOGIA DE ÍLMENORRUTILO E STRÜVERITA DO NORDESTE DO BRASIL
}

\author{
MARIA S. ADUSUMILLI*
}

\begin{abstract}
MINERALOGY OF ILMENORUTILE AND STRÜVERTTE FROM NORTHEASTERN BRAZIL. Ilmenorutile and strüverite, respectively frota Icosinho Pegmatite (State of Ceará) and Lira Pegmatite (State of Paraíba), are mineralogically characterized and compared with rutile and tapiolite. They show a solid solution relation with ionic substitution of $3 \mathrm{Ti}^{4+} \leftrightarrow 2(\mathrm{Nb}, \mathrm{Ta})^{5+}+\mathrm{Fe}^{2+}$. Natural samples of tapiolite are known to be tantalous-ferrifetous with little $\mathrm{Nb}$. The analyses of the samples, together with some from literature, are plotted in $\mathrm{Ti}-(\mathrm{Nb}, \mathrm{Ta})-\mathrm{Fe}$ and $\mathrm{Ti}-\mathrm{Nb}-\mathrm{Ta}$ ternary diagrams, and ( $\mathrm{Nb}, \mathrm{Ta})-\mathrm{Ti}$ binary diagram. They indicate two clear tendencies of solid solution in natural substances, one being of rutile-ilmenonitile (with different proportions of $\mathrm{Ti}: \mathrm{Nb}$ and little Ta), and another of rutile-struverite-tapiolite (where samples of strüverite show a large variation of Ti:Ta and relatively low content of $\mathrm{Nb}$ ). Physical, optical (excluding reflectivity) properties and X-ray diffraction data show general increase hi values ftorn rutile to tapiolite, as a consequence of ionic substitution. Ilmenorutile is inhomogeneous with numerous oriented inclusions identified as evolutions of titanian ferrocolumbite, beside others showing variation in the content of $\mathrm{Ti}$ and $\mathrm{Nb}$, with fixed $\mathrm{Ta}$. Strüverite, though more homogeneous, shows also inclusions with varying $\mathrm{Ti}$ and $\mathrm{Nb}$ content and fixed $\mathrm{Ta}$, one of them identified as titanian tantalite. Though no typical exsolution textures are seen in strüverite, yet the chemical relations of the associated phases suggest exsolution phenomena. The variation in $\mathrm{Ti}$ and $\mathrm{Nb}$ contents in the exsolutions suggest an instability of $\mathrm{Nb}$ in the tetragonal rutile type structure. Structurally, rutile, ilmenorutile and strüverite are mono-rutile type, whereas samples of tapiolite are frequently tri-rutile (ordered) type, but could be also mono-rutile (disordered) type.
\end{abstract}

Keywords: Rutile, ilmenorutile, strüverite, tapiolite, solid solution.

\begin{abstract}
RESUMO Ilmenornitilo e strüverita, respectivamente do Pegmatite Icosinho (Estado do Ceará) e Pegmatite Lira (Estado da Paraíba), são caracterizados mineralogicarnente e comparados com rutilo e tapiolita. Eles mostram uma relação de solução sólida, com substituição de $3 \mathrm{Ti}^{4+} \leftrightarrow 2(\mathrm{Nb}, \mathrm{Ta})^{5+}+\mathrm{Fe}^{2+}$. Amostras naturais de tapiolita são conhecidas por serem tântalo-ferríferas, com pouco $\mathrm{Nb}$. As análises das amostras, junto com algumas da literatura, são plotadas em diagramas temários Ti-(Nb, Ta)-Fe e Ti-Nb-Ta, e diagrama binário (Nb, Ta)-Ti. Estes diagramas indicam duas tendências claras de solução sólida nas substâncias naturais: uma, rutilo - ilmenorrutito (com diferentes proporções de Ti:Nb e pouco Ta); outra, rutilo - strüverita - tapiolita (em que amostras de strüverita têm uma ampla variação Ti:Ta e relativamente baixo teor em $\mathrm{Nb}$ ). Propriedades físicas, ópticas (exclusive refletividade) e dados de duração de raios X mostram, em geral, aumento nos valores de rutilo para tapiolita, como conseqüência da substituição iônica. Ilmenornitilo é heterogêneo com numerosas inclusões orientadas identificadas como exsoluçôes de ferrocolumbita titanífera, além de outras irregulares, mostrando variação no teor em Ti e $\mathrm{Nb}$, com quantidade fixa de Ta. Strüverita, embora mais homogênea, mostra também inclusões com teor variável de $\mathrm{Ti}$ e $\mathrm{Nb}$ e com Ta praticamente invariável, uma delas identificada como tantalita titanífera. Embora não sejam vistas texturas típicas de exsolução em strüverita, as relações químicas das fases associadas sugerem que ocorra este fenômeno. A variação em Ti e $\mathrm{Nb}$ nas exsoluçôes sugere uma instabilidade do $\mathrm{Nb}$ na estrutura tetragonal tipo rutilo. Estruturalmente, rutilo, ilmenorrutilo e strüverita são do tipo mono-rutilo, enquanto tapiolita é mais freqüentemente do tipo tri-rutilo (fase ordenada), mas pode também ocorrer com estrutura mono-rutilo (fase desordenada).
\end{abstract}

Palavras-chaves: Rutilo, ilmenorrutilo, strüverita, tapiolita, solução sólida.

INTRODUÇÃO Os minerais ilmenorrutilo e strüverita foram descritos pela primeira vez, respectivamente, por Kokscharow em 1854, nas montanhas flmen, na Rússia, e por Zambonini em 1907, na Craveggia, na Itália. Têm sido também designados rutilo niobífero ou tantalifero, segundo sugestão de Flinter em 1959 (apua Cerny et al 1964). Parker \& Fleischer (1986) e Fleischer (1987) consideraram válidos os termos originais, os quais serão utilizados neste trabalho.

As espécies guardam entre si uma relação de solução sólida referida como série ilmenorrutilo-strüverita (Cerny et al. 1964). Lima de Faria \& Quadrado (1966) verificaram similaridades e diferenças nos padrões de difração de raios X, bem como variação nas dimensões da cela unitária, na "seqüência rutilo - rutilo niobífero - ilmenorrutilo - strüverita tapiolita", constituindo a série rutilo-tapiolita.

No Brasil, são mencionadas poucas ocorrências de ilmenorrutilo, quase sem destacar localidades e sem qualquer estudo mineralógico: Leonardos (1936) em Minas Gerais; Rolff (1946) em Minas Gerais e na Borborema, no Nordeste, assi- nalando a ausência de strüverita; Abreu (1962) no Maranhão, Ceará, Minas Gerais e Goiás, tratando-se de "rutilo com inclusões de ilmenita"; Silva \& Issa (1988) em Seis Lagos, no Amazonas.

Adusumilli (1976) identificou, entre outros óxidos múltiplos de $\mathrm{Nb}$ e Ta, ilmenorrutilo e strüverita na Província Pegmatítica Nordestina. Com uma complementação de dados por diversos métodos, estas espécies são aqui estudadas mineralogicamente, e sua presença registrada nos Estados do Ceará e Paraíba.

OCORRÊECIA As amostras têm a seguinte procedência: ilmenorrutilo (NT-4, Fotos 1,3 e 5) do Alto do Lira, Paraíba; strüverita (NT-88, NT-91 e NT-101, Fotos 2, 4 e 6) de Icosinho, Ceará; tapiolita do Alto do Onça (NT-2), Alto do Conceição (NT-95), ambos no Estado da Paraíba, e da Serra da Mesa (NT-146), em Goiás. As amostras de rutilo, catalogadas como NT-1, são procedentes de ocorrências pegmatíticas de Goiás e são estudadas somente por propriedades 


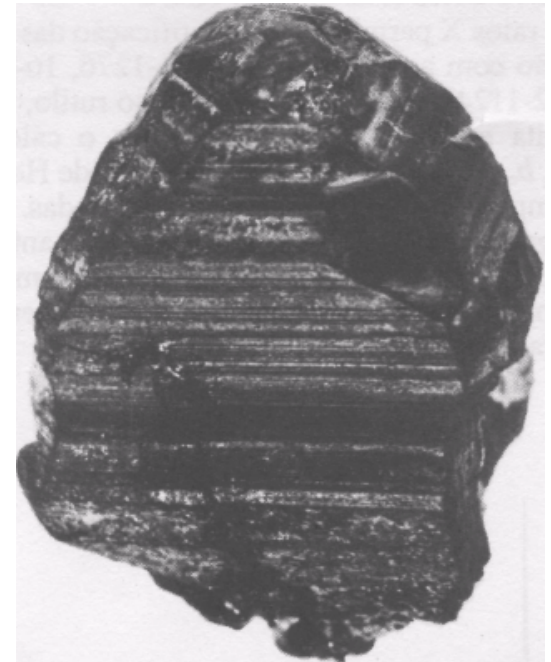

Foto 1 - Cristal de ilmenorrutilo (NT-4), com geminaçao cíclica e estriações características, mostrando à superficie fragmentos defeldspato, quartzo e argilominerais associados. Dimensões: $5,5 \times 4,0 \times 4,5 \mathrm{~cm}$

Photo 1 - Crystal of ilmenorutile (NT-4) with cyclic twinning and characteristic striations, showing fragments of associated feldspar, quartz and clay. Dimensions: $5.5 \times 4.0 \times 4.5 \mathrm{~cm}$

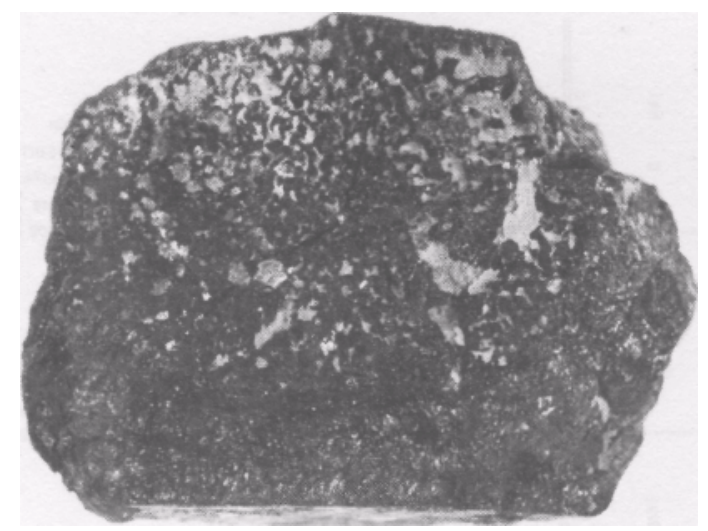

Foto 2 - Cristal tabular espesso de strüverita (NT-101), com delgadas lâminas de muscovita e pequenos grãos irregulares de quartzo efeldspato. Dimensões: $8,0 \times 6,0 \times 3,6 \mathrm{~cm}$ Photo 2 - Thick tabular crystal of strüverite (NT-101) with thin flakes of muscovite and small irregular grams of quartz and feldspar. Dimensions: 8.0 x $6.0 \times 3.6 \mathrm{~cm}$

físicas, morfológicas e radiocristalográficas, com a finalidade de comparação com os outros minerais da solução sólida.

Os pegmatites graníticos mineralizados da província nordestina são geralmente heterogêneos, com os minerais de $\mathrm{Nb}$ e Ta ocorrendo na Zona III, que bordeja núcleos de quartzo leitoso. Na Paraíba, onde se encontram os Altos do Lira, Onça e Conceição, para os quais não se dispõe de descrição, os pegmatites são homogêneos, encaixados em biotita xisto, e de composição variada. Além de quartzo, feldspatos, outros silicates e fosfates, ocorrem freqüentemente minerais de $\mathrm{Nb}$, $\mathrm{Ta}, \mathrm{Be}$ e Li. Em Icosinho, a rocha original é um granito leucocrático; os pegmatites são de pequeno porte, zonados ou não, com associações de ortoclásio (ou microclínio), albita, muscovita, fluorita, berilo e tantalita (Marinho 1967). A formação dos pegmatites é relacionada a um evento geológico de $550 \mathrm{Ma}$, associado às intrusões graníticas. Na Serra da

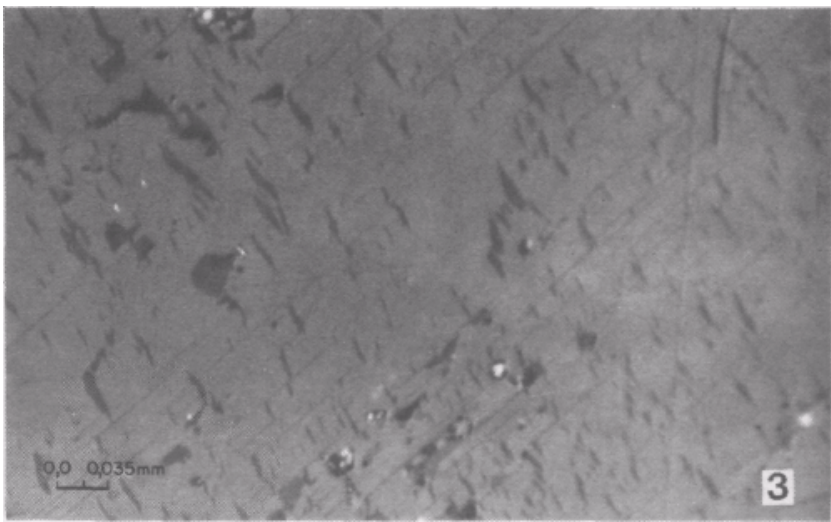

Foto 3 - Secção polida de NT-4, mostrando finas lentes e lamelas orientadas de ferrocolumbita titanifera exsolvida, em matriz de ilmenorrutilo (observações em óleo e nicóis descruzados)

Photo 3 - Polished section of NT-4, showing thin oriented lenses and lamellae of titanian ferrocolumbite exsolved in the matrix of ilmenorutile (Observations in oil, nicols not crossed)

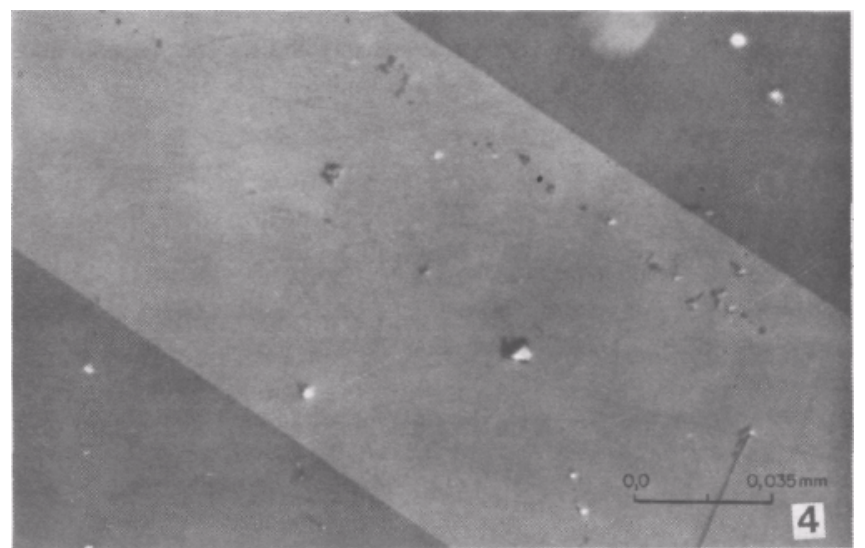

Foto 4 - Secção polida de strüverita geminada, praticamente desprovida de inclusões (observações em óleo e nicóis descruzados)

Photo 4 - Polished section of twinned strüverite, practically without inclusions (Observations in oil, nicols not crossed)

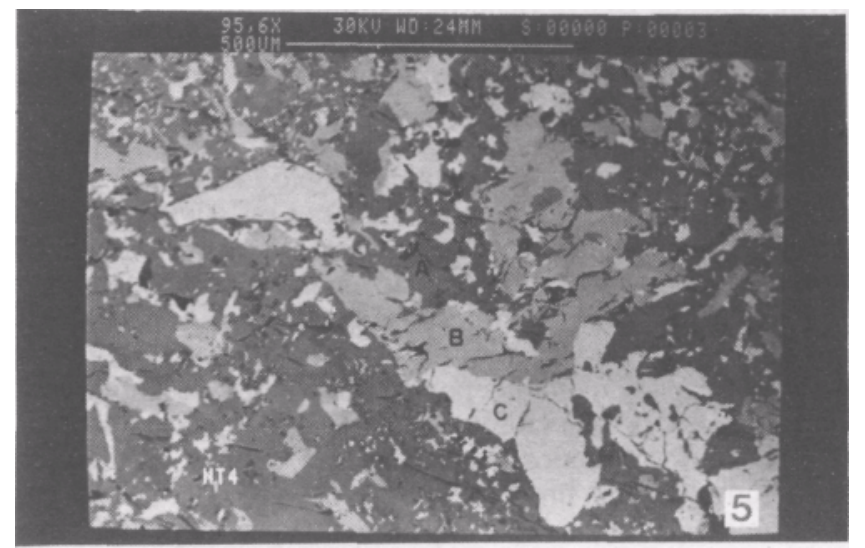

Folo 5 - Aspecto de NT-4 ao microscópio eleírônico de varredura, mostrando numerosas inclusões, entre as quais as fases $4 A, 4 B$ e $4 C$ (simplesmente referidas como $\boldsymbol{A}, \boldsymbol{B}$ e $\boldsymbol{C}$ ) são detectadas pelas diferentes tonalidades da cor cinza $e$ pela composição química variada (Tab. 3)

Photo 5 - Aspect of NT-4 under the scanning electron microscope, showing numerous inclusions, where in phases $4 \mathrm{~A}, 4 \mathrm{~B}$ and $4 \mathrm{C}$ (simply referred as $\mathbf{A}$ $\mathbf{B}$ and $\mathbf{C}$ ) are detected through the different shades of gray colour and variation in chemical composition (Tab. 3) 


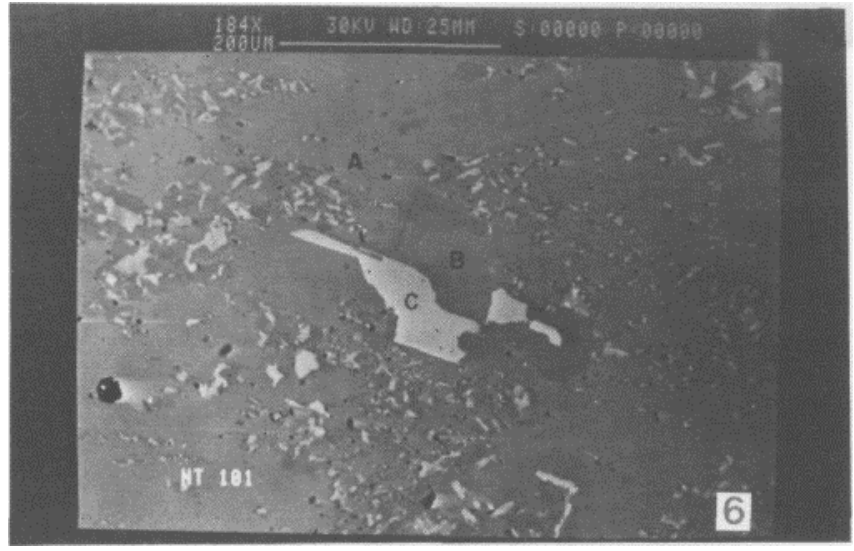

Foto 6 - Microscopia eletrônica de varredura de NT-101 Embora strüverita seja geralmente mais homogênea, é focalizada uma área com inclusões de diferentes dimensões. Três fases distintas $(\boldsymbol{A}, \boldsymbol{B}$ e $\boldsymbol{C})$ com as cores cinza escuro, cinza médio e esbranquiçado, são observadas e analisadas (Tab. 3)

Photo 6 - Scanning electron microscopy of NT-101. Though strüverite is usually more homogeneous, an area with inclusions of different dimensions is focussed. Three distinct phases (A, $\mathbf{B}$ and $\mathbf{C}$ ) with dark gray, medium gray and whitish colours, are distinguished and analized (Tab. 3)

Mesa, Goiás, são conhecidos pegmatites graníticos, onde ocorrem minerais de $\mathrm{Nb}$ e $\mathrm{Ta}$ (columbita-tantalita, ixiolita e tapiolita) e Sn (cassiterita), também pouco estudados.

MÉTODOS DE ESTUDO Os óxidos múltiplos de $\mathrm{Nb}$ e Ta são caracterizados macroscopicamente pela cor, traço, hábitçs comuns e, especialmente, pela densidade de média a alta. E imprescindível a utilização de outros métodos para que se tenha uma identificação precisa das espécies. Amicroscopia óptica de luz transmitida e refletida não fornece dados conclusivos, embora seja muito significativa para indicar aspectos texturais. Métodos radiocristalográficos e o conhecimento da composição química são indispensáveis para a identificação e interpretação de associações e interrelações mineralógicas.

Dessa maneira, a identificação inicial das amostras, após a caracterização macroscópica, foi feita por estudos de raios $\mathrm{X}$ (métodos de Debye-Scherrer e difratometria). Foram utilizados equipamentos Philips e Rigaku, com radiação $\mathrm{CuK} \alpha$, filtro de Ni ou monocromador de grafita, tensão de $40 \mathrm{kV}$ e corrente de $20 \mathrm{~mA}$.

Os métodos ópticos empregados foram especialmente de luz refletida, utilizando-se fotomicroscópio ZEISS III-Pol. São incluídos dados de microdureza Vickers e refletividade (Adusumilli 1976; obtidos com a colaboração do professor W. Uytenbogaardt, então da Universidade Livre de Amsterdam).

Para as análises químicas, foram utilizados diversos métodos:

a. determinação qualitativa por espectrometria de fluorescência de raios X (FRX) (Adusumilli 1976); b. análise quantitativa por vários métodos pelo GEOLAB, GEOSOL, além de duas outras por via úmida (NT-91 e NT-95) já indicadas em Adusumilli (1976); c. análise quantitativa parcial pelo sistema LINK ANALYTICAL AN 10000 por espectroscopia de energia dispersiva (EDS), acoplado a microscópio eletrônico de varredura (MEV) Cambridge S200, com software de aplicação ZAF, no laboratório petrográfico da CBMM, São Paulo (com a colaboração do geólogo Bruno Rippel).

Foi possível, utilizando a referida metodologia, caracterizar mineralogicamente as amostras estudadas, de textura e composição relativamente heterogêneas, bem como estabelecer a correlação com rutilo e tapiolita.
DADOS RADIOCRISTALOGRÁFICOS Os padrões de difração de raios X permitiram a identificação das espécies por comparação com as fichas JCPDS 21-1276, 16-934, 17543, 8-181 (22-1124), respectivamente como rutilo, ilmenorrutilo, strüverita e tapiolita. A indexação e o cálculo dos parâmetros $\mathrm{a}_{0}$, $\mathrm{b}_{0}$ e $\mathrm{c}_{0}$ foram feitos pelo método de Hesse-Lipson e pela comparação com as fichas mencionadas.

Os difratogramas (Fig. 1) são muito semelhantes entre si, como é esperado em substâncias que constituem solução sólida. A comparação dos mesmos, entretanto, permite algumas observações:



Figura 1 - Comparação dos difratogramas de raios $X$ de rutilo, ilmenorrutilo, strüverita e tapiolita, mostrando que há grande semelhança entre eles. Os correspondentes valores de $\boldsymbol{d}$, bem como os parâmetros da cela unitária são levemente diferentes, aumentando de rutilo para tapiolita, como conseqüência da substituição iônica. Alguns picos de columbita (tantalita), indicados pela letra $\boldsymbol{C}$, são observados nos difratogramas de ilmenorrutilo e strüverita, resultado de exsolução. Linhas adicionais no padrão de tapiolita indicam a estrutura tri-rutilo, em vez da mono-rutilo característica das demais espécies

Figure 1 - Comparison of the X-ray diffractograms of rutile, ilmenorutile, striiverite and tapiolite, showing great similarities between them. The corresponding $\mathbf{d}$ values, as well as the unit cell parameters are slightly different, increasing from rutile to tapiolite, as a consequence of ionic substitution. S.ome peaks of columbite (tantalite), indicated by the letter $\mathbf{C}$, are observed in the diffractograms of ilmenorutile and striiverite, result of the exsolution. Additional lines in the pattern of tapiolite indicate the tri-rutile structure, instead of the mono-rutile structure characteristic of the other species 
a. Existe uma leve variação nos valores de $d$ e nos parâmetros unitários, que aumentam de rutilo para tapiolita. As dimensões dos íons $\mathrm{Nb}^{5+}, \mathrm{Ta}^{5+} \mathrm{e} \mathrm{Fe}^{2+}$, principais elementos que substituem o $\mathrm{Ti}^{4+}$ do rutilo, contribuem para o fato.

b. Notam-se linhas adicionais nos espectros de ilmenorrutilo e da strüverita, identificadas pela letra $\mathrm{C}$ (= columbita) na figura 1, cujo aparecimento é relacionado com a presença de columbita ou tantalita exsolvida.

c. Há um maior número de reflexões no padrão de tapiolita, destacando-se as relativas aos planos reticulares 002 e 101, que evidenciam a estrutura tri-rutilo (NT-2 e NT-95). Estudos recentes da amostra NT-146 não mostram tais reflexões, admitindo-se que se trata de uma tapiolita desordenada, com estrutura mono-rutilo.

$E$ É interessante assinalar que certos aspectos morfológicos, como o tipo de fratura, têm sido relacionados com a homogeneidade ou heterogeneidade de ilmenorrutilo (Lima de Faria \& Quadrado 1966). Foi possível também observar, aqui, que fragmentos retirados da amostra NT-4, com fratura conchoidal, não apresentam nos radiogramas as reflexões de columbita, devendo, portanto, ser mais homogêneos. Os grãos com aspecto microgranular ou fratura irregular produzem radiogramas com tais reflexões, sendo assim considerados pertencentes a áreas mais heterogêneas da amostra.

COMPOSIÇÃO QUÍMICA Observações prévias de seções polidas indicaram que algumas amostras são relativamente heterogêneas, contendo mais de uma fase mineralógica (NT-4 e NT-101). Isto, de certo modo, orientou a escolha do método a ser empregado para análise. NT-4, a mais heterogênea, foi analisada por todos os métodos, por se tratar da única amostra de ilmenorrutilo.

Os resultados obtidos por FRX são indicados na tabela 1 , destacando-se a ausência de Bi na amostra NT-91, fato importante para as observações gerais sobre a análise desta amostra na tabela 2 .

Tabela 1 - Composição química por FRX Table 1 - Chemical composition by XRF

\begin{tabular}{|c|c|c|c|}
\hline & NT4 & NT-91 & NT-2 \\
\hline Elemento & $\mathbf{T}, \mathbf{N} \mathbf{b}, \mathbf{T h}, \mathrm{Fe}$ & $\mathbf{T}, \mathbf{T}, \mathbf{F}, \mathbf{N b}$ & $\mathrm{Th}, \mathrm{Fe}, \mathrm{Mb}, \mathrm{Mn}$ \\
\hline Elemento: & Sn & St & $\mathrm{Sn}_{\mathbf{n}}, \mathbf{n}, \mathbf{z}$ \\
\hline
\end{tabular}

Dados quantitativos de composição química são incluídos na tabela 2. Utilizando-se o programa MINFILE, foram calculadas as fórmulas dos minerais e as razões entre os elementos mais significativos para a caracterização dos termos da solução sólida. Com o mesmo programa foram elaborados os diagramas.

A tabela 3 apresenta resultados com porcentagem em peso dos elementos por $\mathrm{MEV} / \mathrm{EDS}$, para três fases nitidamente observadas: 4A, 4B, e 4C em ilmenorrutilo; e 101A, 101B e $101 \mathrm{C}$, em strüverita. Incluem-se também dados calculados dos óxidos correspondentes, bem como as fórmulas químicas e as razões entre os elementos. Nota-se o baixo teor em Ti nas fases columbita (4C) e tantalita (101C). Cerny \& Ercit (1985) chamam atenção para a presença de elementos como Ti, Sn, Sc, W e outros em minerais semelhantes a columbita desordenada, que caracterizam, na realidade, variedades de ixiolita. Considerando o baixo teor de Ti nas fases mencionadas, considera-se que elas referem-se a columbita (ou tantalita) titanífera e não propriamente a ixiolita.

Nas figuras 2, 3 e 4, são apresentados pontos representativos das amostras cujas análises constam das tabelas 2 e 3 . Com a finalidade de melhor fundamentar algumas conclusões e interpretações, são adicionadas análises coletadas na literatura: ilmenorrutilo e strüverita de Cerny et al (1964) e Cerny et al (1981), além de ratilo de Rondônia (Oen et al 1982).
Tabela 2 - Análises químicas e cálculo de fórmulas químicas e razões atômicas de ilmenorrutilo (NT-4), strüverita (NT-88, NT-101, NT-91) e tapiolita (NT-95, NT-146)

Table 2 - Chemical analyses and calculated formulae and atomic ratios of ilmenorutile (NT-4), struverite (NT-88, NT-101, NT-91) and tapiolite (NT-95, NT-146)

\begin{tabular}{|c|c|c|c|c|c|c|}
\hline \multicolumn{7}{|c|}{ S etn peos oxido dos principais elementos } \\
\hline & $\mathbf{N T - 4}$ & Nr-88 & NT-101 & $\mathbf{N T}-91 *$ & NT-95 $\div$ & NT=146 \\
\hline 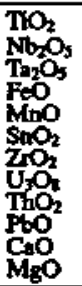 & $\begin{array}{l}39,40 \\
33,80 \\
9,40 \\
12,70 \\
0,08 \\
0,66 \\
0,04 \\
0,13 \\
0,06 \\
0,07\end{array}$ & $\begin{array}{r}22,40 \\
7,40 \\
45,60 \\
13,60 \\
0,22 \\
1,10 \\
0,02 \\
0,01 \\
0,37 \\
0,52 \\
0,03\end{array}$ & $\begin{array}{r}23,50 \\
7,70 \\
48,90 \\
14,50 \\
0,20 \\
1,20 \\
0,04 \\
0,32 \\
0,09 \\
0,00\end{array}$ & $\begin{array}{r}31,60 \\
9,10 \\
35,30 \\
11,52 \\
0,49 \\
0,31 \\
= \\
= \\
= \\
=\end{array}$ & $\begin{array}{l}1,46 \\
0,66 \\
79,81 \\
13,95 \\
1,08 \\
0,95 \\
0,11 \\
0,01 \\
= \\
= \\
=\end{array}$ & $\begin{array}{r}0,29 \\
5,00 \\
7,80 \\
12,50 \\
1,20 \\
0,15 \\
=0,08 \\
0,36 \\
0,04\end{array}$ \\
\hline \multicolumn{7}{|c|}{ Oxidos dos elementos oomponentes de fmpurezss } \\
\hline $\begin{array}{l}\mathrm{N}_{2} \mathrm{O} \\
\mathrm{KO}_{2} \mathrm{O} \\
\mathrm{A}_{2} \mathrm{O}_{3} \\
\mathrm{~S}_{2} \\
\mathrm{P}_{2} \\
\mathrm{TR}_{2} \mathrm{O}_{3} \\
\mathrm{~B}_{2} \mathrm{O}_{3} \\
\mathrm{H}_{2} \mathrm{O}\end{array}$ & $\begin{array}{l}- \\
0,06 \\
0,77 \\
0,71 \\
0,16 \\
0,10 \\
1,93\end{array}$ & $\begin{array}{l} \\
0,22 \\
0,70 \\
6,20 \\
0,13 \\
0,04 \\
1,36\end{array}$ & $\begin{array}{l}- \\
0,02 \\
0,56 \\
1,20 \\
0,10 \\
0,02 \\
1,23\end{array}$ & $\begin{array}{l}\overline{5} \\
0,46 \\
0,66 \\
0,255 \\
6,98\end{array}$ & $\begin{array}{l}\overline{-} \\
\overline{1}, 55 \\
0,32 \\
\bar{y} \\
\overline{0}, 95\end{array}$ & $\begin{array}{l}0,04 \\
\overline{-} \\
0,91 \\
\overline{0}, 11 \\
\overline{0}, 56\end{array}$ \\
\hline Total & 100,07 & 99,92 & 99,64 & 99,675 & 100,00 & 99,42 \\
\hline \multicolumn{7}{|c|}{$\%$ des principais oxidon reculeulados part somi 100,00} \\
\hline 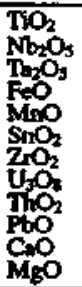 & $\begin{array}{c}40,90 \\
35,08 \\
9,76 \\
13,18 \\
0,08 \\
0,69 \\
0,05 \\
0,13 \\
0,06 \\
0,07\end{array}$ & $\begin{array}{r}24,54 \\
8,11 \\
49,95 \\
14,90 \\
0,24 \\
1,21 \\
0,02 \\
0,01 \\
0,41 \\
0,07\end{array}$ & $\begin{array}{r}24,36 \\
7,98 \\
50,69 \\
15,03 \\
0,21 \\
1,24 \\
0,04 \\
0,33 \\
0,09 \\
0,02\end{array}$ & $\begin{array}{r}34,60 \\
9,96 \\
38,66 \\
15,90 \\
0,54 \\
0,34 \\
= \\
= \\
= \\
=\end{array}$ & $\begin{array}{l}1,49 \\
0,67 \\
81,41 \\
14,23 \\
1,10 \\
0,97 \\
0,11 \\
0,01 \\
= \\
= \\
-\end{array}$ & $\begin{array}{r}0,30 \\
5,11 \\
79,57 \\
13,19 \\
1,23 \\
0,15 \\
\overline{0,04} \\
5,37 \\
\overline{0}, 04\end{array}$ \\
\hline Total & 100,00 & 100,00 & 100,00 & 100,00 & 100,00 & 100,00 \\
\hline \multicolumn{7}{|c|}{ Alomos na fómuln com bese no conteudo de: } \\
\hline & \multicolumn{4}{|c|}{$40^{2}$} & $120^{4}$ & $40^{2}$ \\
\hline 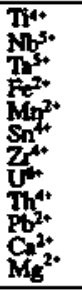 & $\begin{array}{l}1,03 \\
0,53 \\
0,09 \\
0,37 \\
0,00 \\
0,01 \\
=0,00 \\
0,00 \\
0,00 \\
0,00\end{array}$ & $\begin{array}{l}0,70 \\
0,16 \\
0,48 \\
0,53 \\
0,01 \\
0,02 \\
0,00 \\
0,00 \\
0,00 \\
0,03 \\
0,00\end{array}$ & $\begin{array}{l}0,78 \\
0,15 \\
0,59 \\
0,53 \\
0,01 \\
0,02 \\
0,00 \\
0,00 \\
0,00 \\
0,00\end{array}$ & $\begin{array}{l}1,0 \\
0,7 \\
0,11 \\
0,51 \\
0,02 \\
0,01 \\
= \\
= \\
= \\
=\end{array}$ & $\begin{array}{l}0,18 \\
0,06 \\
3,69 \\
1,98 \\
0,15 \\
0,06 \\
0,00 \\
0,00 \\
= \\
= \\
=\end{array}$ & $\begin{array}{l}0,01 \\
0,13 \\
1,19 \\
0,61 \\
0,06 \\
0,00 \\
0,00 \\
0,01 \\
0,01 \\
0,00\end{array}$ \\
\hline Tolat & 2,03 & 2,10 & 2,09 & 2,12 & 6,13 & 2,01 \\
\hline $\mathbf{0}^{-2}$ & 4,00 & 4,00 & 4,00 & 4,00 & 12,00 & 4,00 \\
\hline \multicolumn{7}{|c|}{ Rayōes atōnicas úgnifieativas } \\
\hline $\begin{array}{l}\text { Nb/Ta } \\
\text { Ti/Nb } \\
\text { Ty/T } \\
\text { T/Fe }\end{array}$ & $\begin{array}{l}3,07 \\
1,00 \\
3,07 \\
2,39\end{array}$ & $\begin{array}{l}0,14 \\
2,60 \\
0,36 \\
1,27\end{array}$ & $\begin{array}{l}0,13 \\
2,62 \\
0,35 \\
1,25\end{array}$ & $\begin{array}{l}0,22 \\
2,98 \\
0,66 \\
1,68\end{array}$ & $\begin{array}{l}0,01 \\
1,90 \\
0,01 \\
0,08\end{array}$ & $\begin{array}{l}0,06 \\
0,05 \\
0,00 \\
0,02\end{array}$ \\
\hline
\end{tabular}

- As terras taras (TR) são incluídas entre as impurezas, porque, tendo sido determinadas como TR total, não podem ser consideradas para o cálculo das fórmulas - $\mathrm{O}$ alto teor de $\mathrm{SiO}_{2}$ em NT-88 pode ser atribuído à presença de quartzo, feldspato, muscovita e caolinita associados

- A presença de $\mathrm{BiO}_{3}$ em NT-91 não tem justificativa, podendo ser atribuída a contaminação (bismutotantalita havia sido analisada no mesmo lote) ou a interpretação incorreta. Conforme verificado na tabela 1 , o elemento Bi não foi identificado por FRX

- Há um excesso no total de cations nas fórmulas, o que é conseqüência da substituição heterovalente.

Observations:

- The rare earths (TR) are included as impurities, because they were analyzed as total rare earths. Às such, they could not be included in the calculation of the formulae

- The high content of $\mathrm{SiO}_{2}$ in NT-88 is attributed to the presence of associated quartz, feldspar, muscovite and kaolin

- The presence of $\mathrm{Bi}_{2} \mathrm{O}_{3}$ in NT-91 cannot be explained. It may be attributed to contamination (bistnutotantalite was analised in the same lot) or incorrect interpretation. According to Table $1, \mathrm{Bi}$ was not detected in this sample.

- Análises por L. Favato e F. Peixoto (Minerações Brasileiras Reunidas, Belo Horizonte)

- Analysed by L. Favato and F. Peixoto (Minerações Brasileiras Reunidas, Belo Horizonte) 
Tabela 3 - Resultados quantitativas parciais obtidos por $M E V / E D S$

Table 3 - Partial quantitative results obtained by SEM/EDS

\begin{tabular}{|c|c|c|c|c|c|c|}
\hline \multicolumn{7}{|c|}{ S etp peso } \\
\hline & NT-4A & NT-4B & NT-4C & Mr-JOLA & NI-101B & NI-101C \\
\hline$T^{4+}$ & 32,88 & 15,79 & 4,62 & 17,69 & 21,03 & 2,34 \\
\hline $\mathrm{Nu}^{5+}$ & 15,46 & 28,43 & 42,10 & 8,27 & 5,94 & 22,93 \\
\hline $\mathrm{Ta}^{3+}$ & 6,49 & 6,18 & 5,19 & 32,44 & 32,79 & 35,92 \\
\hline $\mathrm{Fe}^{2+}$ & 8,45 & 14,36 & 12,88 & 10,91 & 10,89 & 5,78 \\
\hline $\mathrm{Mh}^{2+}$ & nnd. & ned & nd. & 0,28 & 0,03 & 7,44 \\
\hline Total & 63,28 & 6476 & 64,79 & 69,59 & 70,68 & 74,41 \\
\hline \multicolumn{7}{|c|}{ 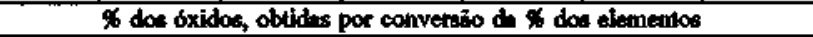 } \\
\hline $\mathrm{TO}_{2}$ & 54,84 & 26,34 & 7,71 & 29,51 & 35,08 & 3,90 \\
\hline $\mathrm{Miby}$ & 22,12 & 40,67 & 60,23 & 11,83 & 8,50 & 32,80 \\
\hline $\mathrm{Tr}_{\mathrm{a}} \mathrm{O}$ & 7,92 & 7,55 & 6,34 & 38,61 & 40,04 & 43,86 \\
\hline Feo & 10,87 & 18,47 & 1657 & 14,04 & 14,01 & 7,44 \\
\hline MhO & $=$ & $\bar{z}$ & $=$ & 0,36 & 0,04 & 9,61 \\
\hline Total & 95,76 & 93,03 & 90,84 & 95,35 & 97,66 & 97,61 \\
\hline \multicolumn{7}{|c|}{ Atomot has finmulat com bate to conteido de: } \\
\hline & \multicolumn{2}{|c|}{$40^{-\frac{2}{2}}$} & $24 \sigma^{2}$ & \multicolumn{2}{|c|}{$4 \mathrm{O}^{-2}$} & $24 \sigma^{2}$ \\
\hline $\mathrm{T}^{+4+}$ & 1,35 & 0,75 & 1,42 & 0,92 & 1,04 & 0,81 \\
\hline $\mathbf{M} \mathbf{b}^{3+}$ & 0,39 & 0,69 & 6,68 & 0,27 & 0,15 & 4,09 \\
\hline $\mathrm{TB}^{\mathbf{3}}$ & 0,07 & 0,08 & 0,42 & $\overline{0,45}$ & $\mathbf{0 , 4 3}$ & 3,29 \\
\hline $\mathrm{Fe}^{2+}$ & 0,30 & 0,58 & 3,40 & 0,49 & 0,46 & 1,71 \\
\hline $\mathrm{Mn}^{2+}$ & - & $=$ & - & 0,01 & 0,00 & 2,24 \\
\hline Tobl & 2,05 & 2,10 & 2,10 & 11,92 & 2,09 & 12,13 \\
\hline$\sigma^{-1}$ & 4,00 & 4,00 & 4,00 & 24,00 & 4,00 & 24,00 \\
\hline \multicolumn{7}{|c|}{ Renbel Alönicas } \\
\hline $\mathrm{Nb} / \mathrm{T}$ & 2,38 & 4,60 & 4,60 & 8,11 & 0,18 & 0,64 \\
\hline NITh & 5,07 & 2,56 & 2,56 & 0,89 & 0,64 & 0,07 \\
\hline TlNb & 2,13 & 0,56 & 0,56 & 0,11 & 3,54 & 0,10 \\
\hline Ti/Fe & 3,89 & 1,10 & 1,10 & 0,36 & 1,93 & 0,40 \\
\hline
\end{tabular}

Nas figuras, as amostras de tapiolita são, como é esperado, tipicamente tantalíferas com apreciável teor em Fe, e ilmenorrutilo e strüverita ocupam campos bem distintos (Fig. 2). Isso leva a considerar que a solução sólida rutilo-tapiolita pode, na realidade, ser expressa de duas maneiras: a. rutiloilmenorrutilo, com larga substituição de Ti por $\mathrm{Nb}$ e pouca variação nos teores de Ta e Fe; b. rutilo-strüverita-tapiolita, com ampla substituição de Ti por Ta e pouca variação dos teores de $\mathrm{Nb}$ e Fe.

PROPRIEDADES GERAIS Lima de Faria \& Quadrado (1966) consideram ilmenorrutilo e strüverita como termos intermediários de uma solução sólida rutilo-tapiolita.

A similaridade dos dados cristalográficos e cristaloquímicos nestas espécies favorece a ocorrência da solução sólida heterovalente entre elas, dentro de amplos limites. Rutilo tem fórmula geral $\mathrm{AO}_{2}$, com $\mathrm{Ti}^{4+}$ como elemento essencial na posição estrutural A e uma estrutura padrão conhecida como mono-rutilo. Ilmenorrutilo e strüverita têm fórmula e estrutura semelhantes às do rutilo, mas o $\mathrm{Ti}^{4+}$ (raio iônico $=0,68 \AA$ ) é substituído por $\mathrm{Nb}^{5+}(\mathrm{r} . \mathrm{i}=0,69), \mathrm{Ta}^{5+}($ r.i. - 0,68$) \mathrm{e} \mathrm{Fe}^{2+}$ (r.i. $=0,74)$ em maior ou menor escala. Tapiolita ocorre segundo duas fases polimórficas: uma, desordenada, $\mathrm{AO}_{2}$, com Ta e Fe predominando na posição A, e estrutura mono-rutilo.; outra, ordenada $\mathrm{AB}_{2} \mathrm{O}_{6}$, com estrutura tri-rutilo, o elemento Ta predominando sobre $\mathrm{Nb}$ no lugar $\mathrm{B}$ e o $\mathrm{Fe}$ predominando sobre $\mathrm{Mn}$ na posição A. Todas têm grupo espacial $\mathrm{P} 4_{2} / \mathrm{mnm}$ e conteúdo da cela unitária $Z=2$. Os raios iônicos idênticos ou com valores próximos e as valências iguais ou diferentes entre os íons que se substituem têm grande significação cristaloquímica. Justificam e contribuem não só para a solução sólida ampla entre as diversas espécies, como pela ocorrência dos dois polimorfos de tapiolita.

$\mathrm{Na}$ tabela 4, tem-se um estudo comparativo das propriedades de rutilo, ilmenorrutilo, strüverita e tapiolita. Os dados de microdureza e refletividade de rutilo foram coletados

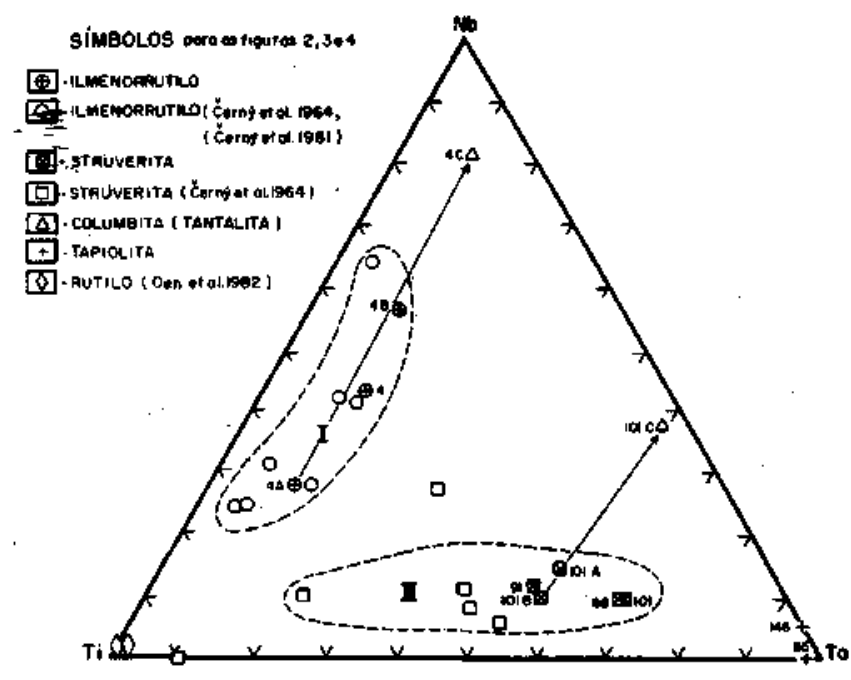

Figura 2 - Variação de composição em diagrama ternário de Ti-Nb-Ta, mostrando a tendência de duas soluções sólidas: a. série rutilo-ilmenorrutilo, com a concentração dos termos titano-niobíferos e pouca variação do teor em Ta, na área I; b. série rutilo-tapiolita, em que os termos titano-tantalíferos, com pouco Nb, ocupam a área II. Duas amostras de strüverita são localizadas fora desta área: uma muito rica em Ti, com pouco Ta e sem $\mathrm{Nb}$, sendo considerada um rutilo tantalifero; outra com acentuado teor em $\mathrm{Nb}$. Os pontos representativos das fases coexistentes na amostra NT-4 (4A, 4B e 4C) e em NT-101 (101 A, 101B e 101C) ficam sobre linhas retas, denotando um enriquecimento em $\mathrm{Nb}$ e decréscimo em Ti no sentido da seta; em ambos os casos, Ta é invariável A correlação química das fases sugere a ocorrência de exsolução de columbita em ilmenorrutilo e tantalita em strüverita

Figure 2 - Variation of composition in ternary diagram of Ti-Nb-Ta, showing the tendency of two solid solutions: a. rutile-ilmenorutile series, with the concentration of titanium-niobiferous terms and little variation in the content of Ta, in area I, b. rutile-tapiolite series, in which titanium-tantaliferous terms, with little $\mathrm{Nb}$, occupy the area II. Two samples of striiverite are recorded beyond this area: one very rich in $\mathrm{Ti}$, with little $\mathrm{Ta}$ and without $\mathrm{Nb}$, considered as tantaliferous rutile; another with accentuated content in $\mathrm{Nb}$. The representative points of the coexistent phases in the sample NT-4 (4A, 4B and 4C) and in NT-101 (101A, 101B and 101C) lie on straight lines, denoting an enrichment in $\mathrm{Nb}$ and decrease in $\mathrm{Ti}$ following the arrow; in both cases, $\mathrm{Ta}$ is invariable. The chemical correlation of the phases suggests the occurrence of exsolution of columbite in ilmenorutile, and tantalite in striiverite

de Uytenbogaardt \& Burke (1971). Foi observada uma variação de propriedades, quase sempre relacionada com a substituição do $\mathrm{Ti}$ do rutilo por $\mathrm{Nb}$, Ta e Fe nas demais espécies, tais como: decréscimo da transparência; cores e traços mais escuros; aumento da densidade; aumento do valor dos parâmetros $A_{o}$ e $\mathrm{C}_{0}$, verificando-se que $C_{0}$ tem valor triplicado no polimorfo ordenado de tapiolita; $\mathrm{Nb} / \mathrm{Ta}$ maior que 1 em ilmenorrutilo e menor que 1 em strüverita e tapiolita; decréscimo da razão $\mathrm{Ti} / \mathrm{Nb}$ de strüverita para ilmenorrutilo e de Ti/Ta de ilmenorrutilo para strüverita; Ti/Fe maior que 1 em ilmenorrutilo e strüverita, e extremamente baixa em tapiolita.

CONSIDERAÇÕES FINAIS O rutilo é um mineral comum, ocorrendo em vários tipos de rochas: sedimentos, pegmatites e como acessórios de outros magmatitos e metamorfitos. Ilmenorrutilo, strüverita e tapiolita são de ocorrência mais freqüente em pegmatites graníticos, ou como acessórios de outras rochas graníticas e alcalinas; entretanto, não são facilmente identificáveis.

Os pegmatites do Nordeste são de natureza granítica e, muitas vezes, enriquecidos em Nb, Ta, Ti, Sn, TR, U, Be, Li, 


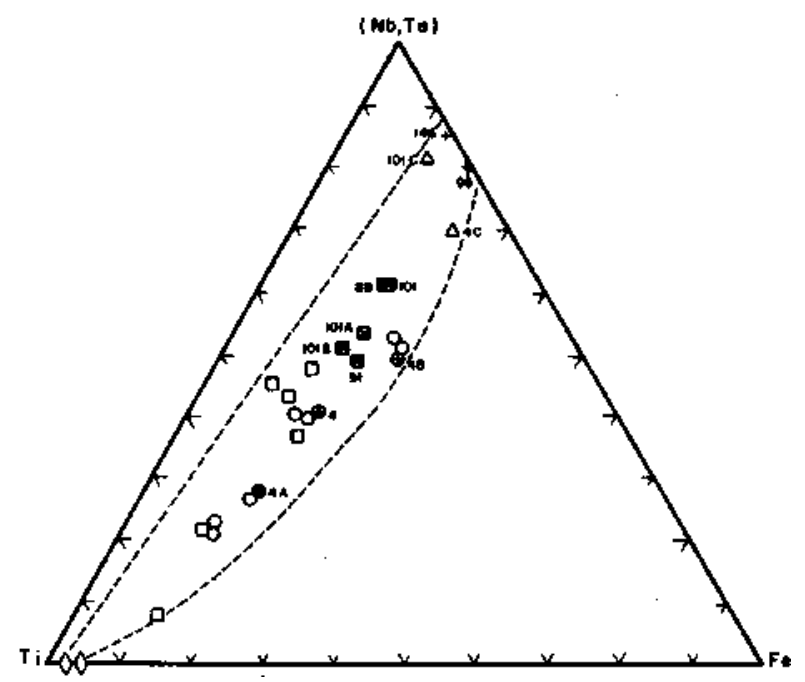

Figura 3 - O diagrama é uma síntese dos diagramas $\mathrm{Ti}$ - $\mathrm{Nb}$ Fe e Ti-Ta-Fe. Dessa maneira, há uma distribuição de pontos como uma típica solução sólida rutilo-tapiolita, sem áreas separadas para a série rutilo-ilmenorrutilo e rutilo-strüveríta-tapiolita. Há um baixo teor em Ti nas fases exsolvidas (columbita e tantalita, respectivamente 4C e 101C) e nas amostras de tapiolita (95 e 146)

Figure 3 - The diagram is a synthesis of diagram Ti-Nb-Fe and Ti-Ta-Fe. As such, there is a distribution of the points as a typical solid solution rutiletapiolite, without separated areas for the series rutile-ilmenorutile and rutile-striiverite-tapiolite. There is a low content in $\mathrm{Ti}$ in the exsolved phases (columbite and tantalite, respectively $4 \mathrm{C}$ and $101 \mathrm{C}$ ) and in the samples of tapiolite (95 and 146)

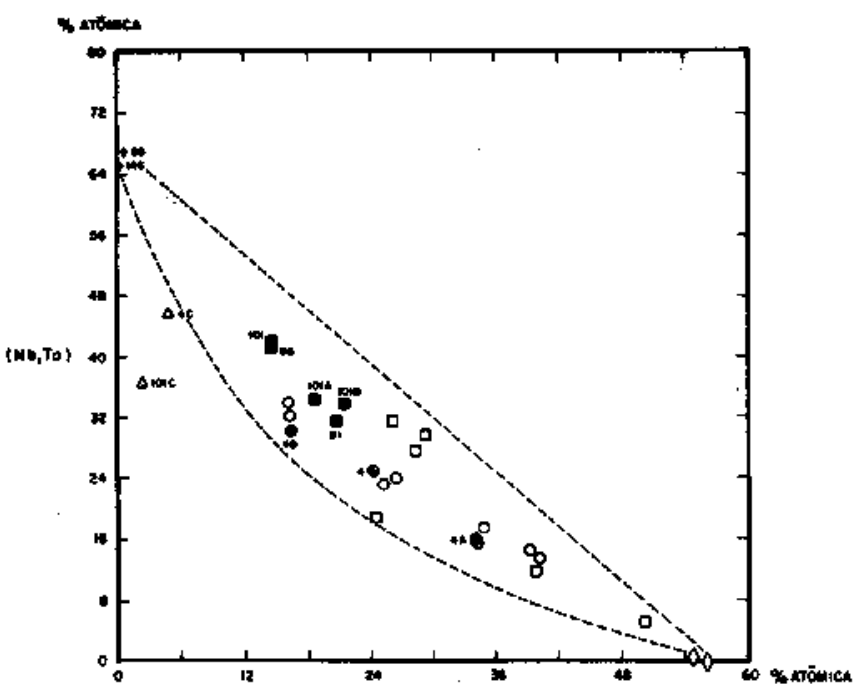

Figura 4 - Distribuição das composições de rutih, ilmenorrutilo, strüverita e tapiolita em Junção da variação ( $\mathrm{Nb}, \mathrm{Ta})-\mathrm{Ti}$, Como na figura 3, este diagrama é uma síntese de dois outros: $\mathrm{Nb}$-Ti e Ta-Ti. Não há áreas especificas para as amostras de ilmenorrutilo e strüverita. Columbita (4C) e tantalita (101C) exsolvidas ficam fora do campo de distribuição, indicando a variação de composição, além da cristalização ortorrômbica em vez de tetragonal

Figure 4 - Distribution of the compositions of rutile, ilmenorutile, striiverite and tapiolite as a function of the variation $(\mathrm{Nb}, \mathrm{Ta})-\mathrm{Ti}$. As in the figure 3 , this diagram is a synthesis of two others: $\mathrm{Nb}-\mathrm{Ti}$ and $\mathrm{Ta}-\mathrm{Ti}$. There is no specific area for the samples of ilmenorrutile and striiverite. Exsolved columbite (4C) and tantalite (101C) are plotted out of the field of distribution, indicating a variation in the composition, besides the orthorrombic crystallization instead of tetragonal

Tabela 4 - Propriedades observadas em ilmenorrutilo e strüverita em comparação com as de rutilo e tapiolita Table 4 - Observed properties of ilmenorutile and striiverite on comparison with the ones of rutile and tapiolite

\begin{tabular}{|c|c|c|c|c|}
\hline & NT-1 & NT4 & Ni-83, NT-101, NT-91 & NT-2, NT-95, NT-146 \\
\hline & RUTILO & ILMENORKUTLO & STRUVERTA & TAPJOLIX \\
\hline 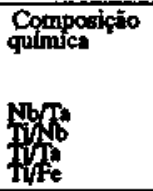 & 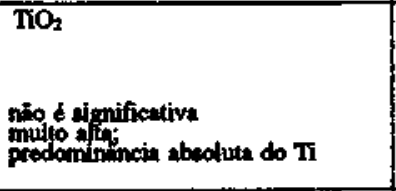 & $\begin{array}{l}\text { (Ti, Nh, Fe, Th) } \mathrm{O}_{2} \\
\text { alta; }>1,5 \\
\text { muito altim; }>2\end{array}$ &  &  \\
\hline Mactescopta & 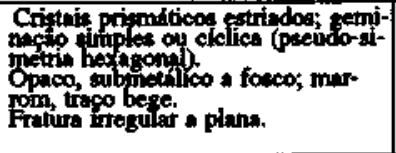 & 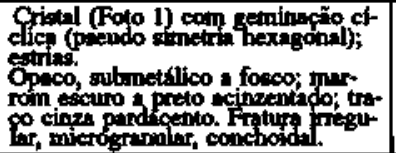 & 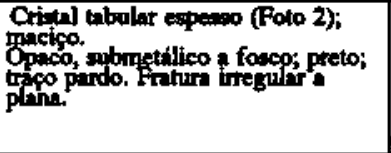 & 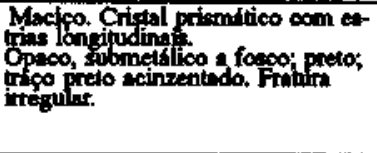 \\
\hline Denginde & $4,18-4,25$ & 4,67 & 5,60 (NT-91) & $7,00=7,88$ \\
\hline Micredeopia & 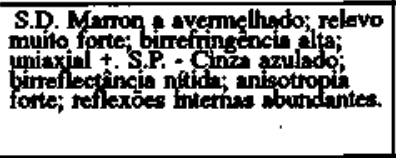 & 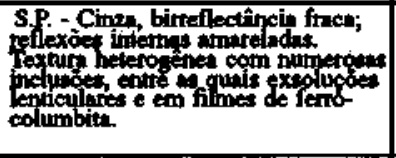 & 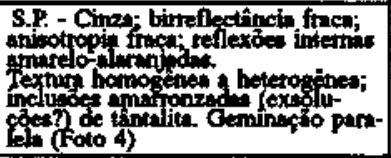 & 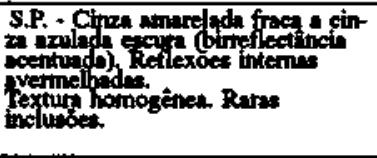 \\
\hline $\begin{array}{l}\text { VFN } \\
\text { kgtmm }\end{array}$ & $933 \cdot 1280$ & $947-1000$ & $995-1059$ & $1011=1039$ \\
\hline $\begin{array}{l}\text { Reflefivida- } \\
\text { de (s) } \\
\text { Rad (nm) } \\
470 \\
589 \\
680\end{array}$ & 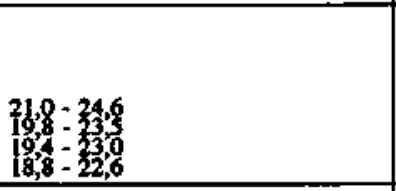 & $\begin{array}{l}20,35=20,65 \\
19,27=29,85 \\
18,67=19,60\end{array}$ & $\begin{array}{l}19,27=28,65 \\
17,77=19 ; 86 \\
17,33-19,50\end{array}$ & $\begin{array}{l}16,36=16,38 \\
13,65=17,16 \\
15,17=16,74\end{array}$ \\
\hline 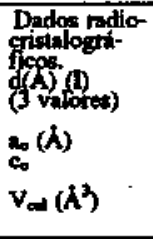 & $\begin{array}{l}3,24 \text { (108) } \\
7,59 \\
2,95 \\
6,4\end{array}$ & 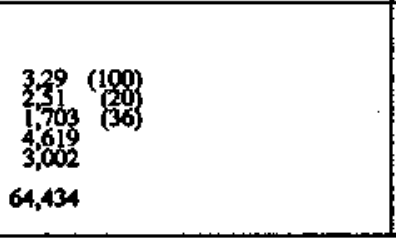 & 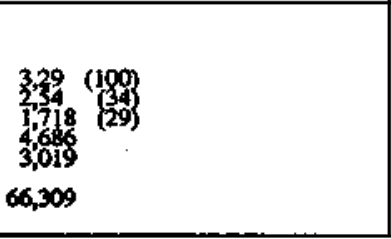 & 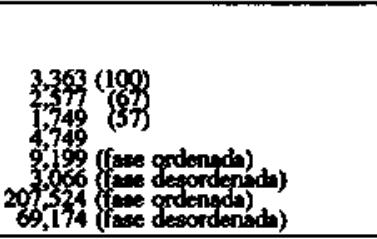 \\
\hline
\end{tabular}


etc. Uma variedade de minerais niobo-tântalo-titaníferos, cristalinos ou metamictos, tem sido identificada. Umenorrutilo e strüverita são registrados e caracterizados neste trabalho.

Com base nos estudos feitos, algumas considerações adicionais podem ser enunciadas:

a. A solução sólida rutilo tapiolita verifka-se com a possível substituição $3 \mathrm{Ti}^{4+} \leftrightarrow 2(\mathrm{Nb}, \mathrm{Ta})^{5+}+\mathrm{Fe}$. Sendo uma substituição entre cations de diferentes valências, as fórmulas calculadas mostram um excesso de cargas positivas em relação às cargas negativas (total de $\mathrm{O}^{2-}$ na cela unitária). Bursill \& Blandim (1984) admitem defeitos estruturais em rutilo pela ocorrência de vazios nas posições de $\mathrm{O}^{2-}$. Tal fato pode ocorrer também, contribuindo para o excesso de cargas positivas.

b. A solução sólida entre os minerais aqui estudados indica claramente a existência de duas tendências para as substâncias naturais: uma, rutilo-ilmenorrutilo, com a substituição predominante de $\mathrm{Ti}$ por $\mathrm{Nb}$; outra, rutilo-strüverita-tapiolita, com a substituição preferencial de Ti por Ta.

c. A maior heterogenidade das espécies niobíferas (ilmenorrutilo) revela, talvez, uma menor afinidade do $\mathrm{Nb}$ com a estrutura tetragonal, pois há numerosas exsoluções de ferrocolumbita titanífera, que têm cristalização ortorrômbica. d. A espécie tantalífera (strüverita), embora mais homogênea, mostra também inclusões, entre as quais é variável o teor em $\mathrm{Ti}$ e Nb, com Ta mais ou menos constante. Entre elas, identificou-se uma tantalita titanífera. $\mathrm{O}$ aspecto textural das diversas fases associadas não indica conclusivamente uma relação de exsolução, que é apenas sugerida pela relação existente na composição química.

e. É desejável um estudo mais detalhado para elucidação da natureza das numerosas fases associadas, especialmente em ilmenorrutilo, o que seria possível com um minucioso estudo por microssonda eletrônica.

f. Finalmente, deve ser mencionada a possibilidade de aproveitamento econômico de ilmenorrutilo e strüverita. dados os teores de $\mathrm{Ti}, \mathrm{Nb}$ e $\mathrm{Ta}$ que apresentam.

Agradecimentos Agradecimentos especiais são feitos ao Conselho Nacional de Desenvolvimento Científico e Tecnológico $(\mathrm{CNPq})$, pelo patrocínio e incentivo às pesquisas; às instituições mencionadas no texto, sem a colaboração das quais, não seria possível obter certos dados experimentais; a Luizhélio Barreto e ao prof. Geraldo Ferreira de Andrade, pela doação de amostras; ao dr. A. Bhaskara Rao e ao dr. Daniel Atencio pelas críticas e sugestões.

\section{REFERÊNCIAS BIBLIOGRÁFICAS}

ABREU, S.F. 1962. Recursos Minerais do Brasil vol. II. Combustiveis fósseis

e minérios metálicos. Rio de Janeiro, Minist. Ind. e Com./Inst. Tecnol. $694 \mathrm{p}$.

ADUSUMILLI, M.P.S. 1976. Contribuição à Mineralogia dos Niobo-tantalatos da Província Pegmatítica Nordestina. Belo Horizonte. 212 p. (Tese de Livre Docência, UFMG).

BURSILL, L.A \& BLANDIM, M.G. 1984. Structure of small oxigen vacancy defects in nonstoichiometric rutile. Jour. Solid State Chem., 51:321-335.

CERNÍ, P.; CHECH, E; POVONDRA, P. 1964. Review of ilmenorutile-strüverite minerals. N. Jb. Miner. Abh., 101(2): 142-172.

CERNtf, P. \& ERCIT, T.S. 1985. Some advances in the mineralogy and geochemistry of $\mathrm{Nb}$ and $\mathrm{Ta}$ in rare-element granitic pegmatite. Bull. Mineral, 108:499-532.

CERNt, P.; PAUL, B.J.; HAWTHORNE, EC.; CHAPMAN, R. 1981. A niobian rutile - disordered columbite intergrowth from the Huron Claim pegmatite, southeastern Manitoba. Can. Mineral, 19:541-548.

FLEISCHER, M. 1987. Glossary of mineral species. 5 ed. Tucson, Mineralogical Record Inc. 227 p.

LEONARDOS, O.H. 1936. Ensaios de concentração de ilmenorrutilo do sul de Minas Gerais. Mineração e Metalurgia, 1(3):91-92.

LIMA DE FARIA, J. \& QUADRADO, R. 1966. Ilmenorutile and strüverite from Nampoça, Alto Ligonha, Mozambique. Garcia Orta, 14:305-310.
MARINHO, J.M.L. 1967. Contribuição ao estudo dos pegmatitos do Ceará. Fortaleza, SUDECO/SEVOME/Dept. de Minas.

OEN, IS · KORPERSHOEK, H R · KIEFT $\mathrm{C}$; LUSTENHOUWER, W J. 1982. A microprobe study of rutile, cassiterite, wolframite and sulfides in the Morro Potosi greisen, Rondônia, Brasil. N. Jb. Miner. Mh., 4:175191.

PARKER, L.R. \& FLEISCHER, M. 1968. Geochemistry of Niobium and Tantalum. Washington, US Geological Survey. 43 p. (Paper 612).

ROLFF, P.M. A. 1946. Minerais de pegmatitos da Borborema. Rio de Janeiro, DNPM. 78:23-76.

SILVA, A.B. \& ISSA, A., F. 1968. Recursos mundiais de nióbio. Nióbio em perspectiva, 3:16 $\mathrm{p}$.

UYTENBOGAARDT, W. \& BURKE, E.A.J. 1971. Tables for microscopic identification of ore minerals. 2nd ed. Amsterdam, Elsevier;430 p.

MANUSCRITO A660

Recebido em 21 de maio de 1990 Revisão do autor em 05 de janeiro de 1991 Revisão aceita em 04 de fevereiro de 1991 